\title{
Progress in the Development of Animal Rabies Vaccines in China
}

\author{
Chunsheng Yin ${ }^{1, *}$
}

Rabies is caused by neurotropic viruses of the genus Lyssavirus in the family Rhabdoviridae, of the order Mononegavirales and can be transmitted to all mammals (1-2). Rabies will infect humans who are bitten or scratched by rabies-infected animals, including dogs, cats, bats, foxes, wolves, rats, weasels, badgers, raccoon dogs, etc. (3-5). At present, there are no effective cures for the disease, and the fatality rate is almost $100 \%$.

Rabies cases have been recorded in China since ancient times as there were recorded in "Huainan $\mathrm{Zi}$ ", telling the story of the rabies dog biting Ziyang, which led to his death and was likely the first recorded case of rabies in Chinese history. Even at present, China is still seriously affected by rabies with more than 200 cases of rabies morbidity and death reported every year $(6-7)$. Infected dogs and cats are the main sources of rabies infections. Strict management of dogs and cats and active immunization are active and effective methods to control rabies transmission among humans. Rabies vaccine development has gone through different stages, including nerve tissue vaccine, avian embryo tissue vaccine, cell culture-derived live virus vaccine, cell culture-derived inactivated virus vaccine, attenuated virus vaccine with reverse genetic manipulation research, nucleic acid vaccine, and recombinant genetic engineering subunit vaccine and live vector vaccine (8).

\section{ANIMAL NERVE TISSUE VACCINE}

In the 1950s, Chinese veterinary scientists developed an attenuated vaccine from sheep brain tissue. Although the vaccine could protect animals' immunity, its residual virulence was still pathogenic to some animals. By the 1960s, China had also used sheep brain tissue to prepare inactivated vaccines. The vaccine was not pathogenic to animals and was safer. However, this vaccine could cause paralysis-like symptoms to animals for the components of goat or sheep tissue. Nevertheless, the vaccine was proved to be effective by using experiment dogs to carry out immunity efficacy tests. The use of the neural tissue inactivated seedlings on early animal rabies prevention and control played an important role. The nerve tissue vaccine had been replaced by other vaccines due to the encephalitis factor related to the myelin in brain tissue, which could trigger allergic encephalomyelitis and cause death, lifelong paralysis, paraplegia, and other adverse consequences.

\section{ATTENUATED VACCINES FOR POULTRY EMBRYO CULTURE}

Avian embryo attenuated vaccine can be prepared by using a virus to infect avian embryos. Flury low egg passage (LEP) virus and Kelev were the main strains for the production of this vaccine. Virus strains were inoculated into Specific Pathogen Free (SPF) chicken embryos, and the allantoic fluid was harvested and lyophilized after virus proliferation. This vaccine was recommended for muscular immunity in dogs, cats, and cattle for a one-year duration of immunity. The most widely used strain of chicken embryo vaccine was the Flury strain. The strain was isolated from human brain tissue from patients who died of rabies in 1940 and was passed in the brain of one-day-old chickens for 138 generations, and then passed through chicken embryos for 40-50 generations. After passing through chicken embryos for more than 178 generations, the pathogenicity to animals was greatly reduced, but it was still pathogenic to inoculate in the brain of suckling mice and monkeys. The chicken embryo vaccine was used to inoculate rabies virus with chicken embryo, which was easier to operate than the brain tissue vaccine and had no neurological side effects compared to the brain tissue vaccine.

\section{LIVE ATTENUATED VACCINE IN CELL CULTURES}

The LEP strain, Evelyn-Rokitnicki-Abelseth (ERA) strain, CTN-1 strain, and their modified strains were used to produce live attenuated vaccine in cell culture. In China, live rabies vaccine was first used in 1980. In 1977, China Institute of Veterinary Drug Control 
(IVDC) adapted rabies LEP to proliferate in the BHK21 cell line. Results showed that virus growth was stable and the virus content per $0.03 \mathrm{~mL}$ was above $10^{4.0} \mathrm{LD}_{50}$. There were no adverse reactions after intramuscular injection of the lyophilized vaccine in 3month-old dogs and rabbits. Once an intramuscular injection is made on domestic dogs, the immunity period is more than 1 year. The vaccine has been approved for production and put into use since 1980 (9).

In 1983, IVDC introduced the ERA strain of rabies virus from abroad. BHK-21 cells and primary porcine kidney cells were used to replicate the virus, and the biological characteristics were identified. The strain had good immunogenicity and no adverse reactions were observed in dogs, cattle, sheep and other animals. After propagation and passage on $\mathrm{BHK}-21$ cells, the virus content was more than $10^{5.0} \mathrm{LD}_{50} / 0.03 \mathrm{~mL}$. The lyophilized vaccine was made from BHK-21 cells and immunized dogs, horses, cattle and sheep with different doses. The immunization period was more than one year. The vaccine had been tested in 13 provinces, with no adverse reactions. Compared with LEP strain, ERA strain was safer and more suitable for target animals.

At the same time, IVDC had also carried out oral immunization tests with the vaccine in a large number of dogs. Domestic dogs were immunized with different doses of vaccine and feeding methods, which increased the level of safety. Samples were collected at different periods after immunization to detect serum neutralizing antibodies against rabies. The antibody positive conversion rate $(1: 8)$ was $40 \%-50 \%$.

In the following years, IVDC cooperated with a number of biological products manufacturers to develop a live primary cell vaccine of ERA strain for rabies, using gophers, donkeys, sheep, pigs, cattle, and other animals of different origin for the primary cells. The results showed that bovine testis primary cells inoculated with ERA strain had the best adaptability, high yield, and stable virulence. Furthermore, ERA/BT cell adaptive strain of the rabies virus was developed. Bovine testis primary cells were used to produce the vaccine successfully.

However, the attenuated live vaccine also had some potential safety hazards, because the virus may cause disease due to the regurgitated mutation in vivo or it may cause gene rearrangement with the naturally infected epidemic strains producing strains. Therefore, commonly used vaccines were mainly inactivated vaccines rather than attenuated vaccines. China stopped manufacturing and using live rabies vaccine on June 30, 2018 (10).

\section{INACTIVATED VACCINE IN CELL CULTURES}

At present, beta-Propiolactone (BPL) is commonly used to inactivate rabies virus at $4{ }^{\circ} \mathrm{C}$ in China, and formaldehyde is no longer used as the inactivated agent. BPL can destroy viral nucleic acid but does not change the structure of viral protein or affect the antigenicity of rabies virus. The inactivated antigen can be completely hydrolyzed in the vaccine liquid, with no inactivated agent residue in the finished vaccine (10). Therefore, the usage of BPL can avoid the reduction of virus immunogenicity and toxic substances or irritants residue. Virus strains used in the preparation of inactivated vaccines usually include the SAD strain, ERA strain, LEP strain, CVS-11 strain, PM strain, PV strain, etc. Stromal cells were used in the preparation of attenuated vaccines generally with BHK-21, MDCK, Vero, etc. Many kinds of inactivated rabies vaccines were produced and used in China, including the inactivated rabies vaccine (LEP strain) developed by IVDC, the inactivated rabies vaccine (PV2061 strain) developed by Liaoning Cheng Da Animal Pharmaceutical Co., Ltd., the inactivated rabies vaccine (LEP strain) developed by Liaoning Yikang Biological Co., Ltd., the inactivated rabies vaccine developed by PLA Academy of Military Medical Sciences (CVS-11 strains), the inactivated rabies vaccine developed by Tangshan Yian Biological Engineering Co., Ltd. (CTN-1 strains), the inactivated rabies vaccine developed by Changzhou Tongtai Biological Pharmaceutical Technology Co., Ltd. (SAD strains), the inactivated rabies vaccine (DG strain) developed by South China Agricultural University, and the inactivated rabies vaccine (R3G strain) developed by Shandong Huahong Biological Engineering Co., Ltd.

\section{AN ATTENUATED VACCINE DEVELOPED BY REVERSE GENETIC TECHNIQUE}

Reverse genetic technology is cloned by molecular biology technology on the basis of the full genome sequence, through the modification of target genes and mutations to assemble a new individual virus. This platform as the research basis can be used to study the 
influence of gene changes on the phenotype of the organism. Therefore, the development of reverse genetics technology is of great significance to the study of life sciences.

With the application and development of reverse genetics technology, many achievements have been made in the research of live rabies vaccine. To establish the rabies virus reverse genetic operation, the expression of antisense RNA genome expression plasmid (genome plasmid) and three kinds of expressions of virus nucleoprotein protein $(\mathrm{N})$, phosphorus $(\mathrm{P})$, and large transcription protein $(\mathrm{L})$ of plasmid (auxiliary plasmid) should to be established. With cells expressing T7RNA polymerase transfection, the antisense genomic RNA and these $\mathrm{N}, \mathrm{P}$, and $\mathrm{L}$ proteins form an antisense genomic ribonucleoprotein complex (RNP). The antisense genomic RNP complex has the same biological activity as the RNP complex produced in rabies virus-infected cells. Therefore, genomic RNA can be synthesized using antisense genomic RNP as a template, and then mRNA can be synthesized from genomic RNP and expressed viral proteins. Infectious recombinant rabies virus was produced after the assembly of genomic RNP, membrane protein $(\mathrm{M})$, and glycoprotein $(\mathrm{G})$. The mutation of arginine or lysine 333 on $G$ protein plays a decisive role in the pathogenicity of rabies virus. According to the analysis of $G$ protein epitope of rabies virus, the recombinant virus of $\mathrm{G}$ gene from different strains of rabies virus will be derived. The results show that the substitution of this amino acid residue can effectively weaken the virus strain. By inserting another 2 or $3 \mathrm{G}$ genes to construct recombinant virus $\mathrm{G}$ protein stability and expressing them using the original manufacturer's mass traditional methods of $\mathrm{G}$ protein that had similar stability, Chinese scientists have successfully constructed the $\mathrm{dG}$ and $\mathrm{r} 3 \mathrm{G}$ strains virus containing two or three $\mathrm{G}$ proteins (11).

In conclusion, the characteristics of the attenuated live vaccine constructed by reverse genetic manipulation are low production cost and simple production procedure. Due to the characteristics of the attenuated live vaccine, the most concerning issue is the safety of the vaccine. The reason is that after the recombinant virus immunized the animal, once the animal is recessive, the wild strain and the recombinant virus may be recombined, and the original missing genes can be restored through recombination and the original virulence can be regained. In the future, it may be mainly used for oral vaccine immunization in wild animals.

\section{GENETICALLY ENGINEERED RECOMBINANT LIVE VECTOR VACCINE}

Recombination live vector vaccine is prepared by using gene engineering technology to construct virus or bacteria into a vector and insert foreign genes into it to express the constructed live vector. The live vector vaccine has the advantages of both the conventional attenuated live vaccine and the inactivated vaccine. It has the advantages of high immunity efficacy and low cost of the attenuated live vaccine and good safety of the inactivated vaccine.

In rabies live vector vaccine research, poxvirus and adenovirus are researched and used comprehensively as carriers. In 1992, the rabies virus G gene of CVS-11 strain were inserted into a recombinant poxvirus vector to construct recombinant poxvirus through subcutaneous immunization in mice and rabbits and guinea pigs were immunized through oral immunization (12). Immunization with the recombinant poxvirus subcutaneously and in the muscle could induce neutralizing antibodies with high titer, but no positive antibodies were observed by oral immunization. In 1993, Zhao WG et al. also adopted the recombinant poxvirus expression system, and successively constructed the recombinant poxdisease expressing the $\mathrm{N}$ protein of rabies virus and the recombinant virus Tiantan strain of vaccinia virus coexpressing the $\mathrm{G}$ protein and $\mathrm{N}$ protein of CVS-11 strain of rabies virus (13). The immunization of mice showed that the neutralizing antibodies could be produced after immunization, and could resist attack by rabies virus. In $2001, \mathrm{Li} \mathrm{WH}$ et al. constructed a recombinant adenovirus expressing the $G$ protein replication defect of rabies virus (14). After immunizing mice with this virus, the mice were attacked with a lethal amount of rabies virus, and the protection rate of immunized mice reached $87.5 \%-100 \%$. Immunization of target dogs could induce a high level of protective rabies virus specific neutralizing antibody. In 2006, Zhang SF et al. constructed canine adenovirus serotype 2 vector vaccine expressing rabies virus glycoprotein, and the experimental results showed that it could resist the virulent rabies virus challenge with an immune effect similar to that of conventional inactivated vaccine (15). Furthermore, the protective neutralizing antibody remained in immunized dogs for several months, showing the potential to replace the existing vaccine. The results showed that about $87.5 \%$ of the 
immunized dogs produced neutralizing antibodies, which can be detected 2 to 3 weeks after injection, peaking at 5 to 6 weeks later. The serum neutralizing antibody level of $90.8 \%$ dogs was more than 24 months, and the antibody titer was higher than $0.5 \mathrm{IU} / \mathrm{mL}$, showing a gradual but slow decline (16). The recombinant vaccine could be taken orally with a good immunity effect on dogs. Recombinant human adenovirus type 5 expressing $G$ protein of attenuated SRV9 strain of rabies virus was constructed in 2011. The complete open reading frame of G gene of SRV9 strain of rabies virus was cloned into the shuttle plasmid polyclonal site of adenovirus expression system. Overall, $293 \mathrm{AD}$ cells were co-transfected with linearized skeletal plasmid and recombinant shuttle plasmid mediated by Roche's transfection solution. After 14 days, anti-rabies neutralizing antibody was produced, and the effective protection rate reached $90 \%$ (16).

\section{NUCLEIC ACID VACCINE}

Nucleic acid vaccine prepared by gene recombination technology will have a certain immune active antigen gene promoter downstream of restructuring in eukaryotic expression system and constructing the recombinant expression vector, after a large number of extraction of plasmid DNA is injected into animals by subcutaneous, intramuscular injection, or gene gun methods. By the transcription of host cell synthesis, the DNA vaccine stimulates the host's immune system to produce immune response to the protein. The nucleic acid vaccine is characterized by easy operability and construction. In addition, the nucleic acid vaccine can induce immune animals to produce humoral and cellular immune responses. Because the nucleic acid vaccine only produces the corresponding immune response to the designated antigen, but not for other unrelated antigens, it does not affect the use of other vaccines.

In the rabies virus nucleic acid vaccine research, the cDNA of rabies virus $G$ protein was placed downstream of the SV40 promoter, then the constructed plasmid DNA was directly injected into the gastrocnemius muscle of mice and serially immunized 3 times, each time with an interval of 2 to 3 weeks. Anti-rabies virus neutralizing antibody and cellular immune response were produced in mice after immunization. Xiang ZQ et al. combined immunizing mice with murine granulocyte-phage colonystimulating factor and rabies virus DNA vaccine could significantly improve the level of humoral immunity and cellular immune response (17).

The nucleic acid vaccine has a lot of advantages; for example, it is easy to construct and preserve without cold chain transportation, which can make up for the traditional inactivated or attenuated vaccine shortages. However, there are also disadvantages to this kind of vaccine, mainly plasmid DNA in the body for a long time, causing gene mutations or leading to cancer and other potential harm. Given the safety concerns of nucleic acid vaccines, which have yet to be resolved, nucleic acid vaccines cannot replace the vaccines currently in use.

\section{GENETICALLY ENGINEERED SUBUNIT VACCINES}

The genetically engineered subunit vaccine refers to the use of recombinant DNA technology, the coding of pathogenic microorganism protective antigen gene fragments into the prokaryotic or eukaryotic expression vectors, so that the virus protein can be highly expressed, the protective antigen can be extracted and then added to the adjuvant emulsification to prepare the genetic engineering subunit vaccine. The vaccine contains only one or more antigen epitopes of the pathogen, but no other genetic information of the pathogen, and can be used to inoculate animals with these vaccines to obtain protective immunity. Because the subunit vaccine does not contain infectious components, it does not need to be inactivated, nor does it have pathogenicity, so the prepared vaccine has high safety.

$\mathrm{G}$ and $\mathrm{N}$ proteins of rabies virus are the main antigens that induce humoral and cellular immune responses. Therefore, $\mathrm{G}$ protein and $\mathrm{N}$ protein are mainly proteins in the research of rabies subunit vaccine. In 1983, the G gene of CVS-11 strain of rabies virus was inserted into the bacterial expression plasmid, and then transformed into Escherichia coli and successfully expressed $G$ protein. However, due to the lack of glycosylation function in the prokaryotic expression system, the immune effect of the expressed protein was very poor. The $G$ gene of rabies virus $S A D$ strains was inserted into the yeast expression system, with a high protein expressing quantity. However, the main problems existing in the system are that the expression of the type of $\mathrm{G}$ protein glycosylation and the location of the glycosylation are different from the original strain, as there is no correct $G$ protein glycosylation. Therefore, the immune effect of $G$ protein is affected. At present, the $G$ protein of the 
rabies virus was successfully expressed in SF9 cells using baculovirus expression system, and purified it to prepare vaccine to immunize animals. The results showed that the protein could induce the production of protective neutralizing antibodies and resist the lethal dose of the virus after oral immunization (18).

\section{RESEARCH PROGRESS OF ADJUVANT FOR RABIES VACCINE}

Adjuvants can improve the immunogenicity of subunit vaccines, thus reducing the amount of vaccine needed, the cost of vaccine production, and the number of immunization failures. Inactivated vaccines currently in use include either no adjuvant or aluminum adjuvant. Aluminum adjuvant can enhance Th2 response in mice and induce IgG1 antibody production. Tests have shown that the inactivated vaccine containing $\mathrm{Al}(\mathrm{OH})_{3}$ can immunize dogs and cats for up to 3 years. In recent years, scientists have gained a deeper understanding of the basic immune pathways that enhance the immune response and have known that the activation of the innate immune response is the most critical step in the acquired immune response. Cell signaling involved in the activation of innate immune response cells has been well identified, such as Toll-like receptors and NODlike receptors. Therefore, many new adjuvants are developed to replace aluminum adjuvants, such as the new adjuvant $\mathrm{CpG}$ oligo deoxynucleotide, which can bind to TLR-9 to activate the innate immune response. The research team prepared inactivated rabies vaccine with aluminum adjuvant and CpG adjuvant respectively in mice and carried out a comparative test. The antibody level of CpG immunized for 3 times was similar to that of aluminum adjuvant immunized for 5 times. In recent years, many new adjuvants have emerged or have been studied in preclinical trials. Their modes of action have been described in detail in the literature, and it is necessary to develop a second generation of rabies vaccine adjuvants based on these theories.

\section{QUALITY CONTROL OF INACTIVATED RABIES VACCINE FOR ANIMAL USE IN CHINA}

As of December 2020, the Ministry of Agriculture and Rural Affairs of China had approved the new veterinary drug certificate of 9 inactivated rabies vaccines for animals. All live rabies vaccines (including related combined vaccines) have been stopped for animal immunization since June 30, 2018. The quality standard control indexes of inactivated rabies vaccine for animal use in China are not completely consistent, but mainly including the following indexes. The physical properties of the vaccine should conform to the labeling requirements of the product; vaccines should be pure and are usually tested for sterility according to the current edition of the Chinese Veterinary Pharmacopoeia. The inactivation test of the vaccine was usually the number of mice (weight, age, and quantity varied according to different products) inoculated in the brain, $0.03 \mathrm{~mL}$ for each, and observed for 21 days. Some products should be subcutaneously inoculated into mice, $0.5 \mathrm{~mL}$ for each, and observed for 7-14 days. All the above test mice should be healthy, and subcutaneously inoculated mice should not show any reaction at the injection site. The safety test of the vaccine requires intramuscular injection of at least 2 healthy susceptible dogs (usually beagles) with negative antibodies to rabies virus, and subcutaneous inoculation of 1 or 2 doses of vaccine in mice and guinea pigs for 21-28 days. All of test animals should be healthy and alive. The most commonly used test for vaccine efficacy is the National Institutes of Health (NIH) method derived from the $\mathrm{NIH}$, but the specific procedures for the NIH method vary widely from different products. The method is to use the same dose of rabies virus (such as CVS-24 strain), challenge two (or one) intraperitoneal injection of different dilution of the vaccine to be tested and international standard (reference) vaccine. According to the $\mathrm{PD}_{50}$ value of each group, the number of international units of the vaccine to be tested relative to the international standard (reference) vaccine should be calculated. The standard of inactivated rabies vaccine in China is that each dose of vaccine should be $\geq 2$ international units (IU). In the Chinese Veterinary Pharmacopoeia (2020 edition, the third volume appendix 3407), a unified method for testing the efficacy of inactivated rabies vaccine for veterinary has been developed, which reduces the errors of National Institutes of Health test results in different laboratories to a certain extent.

At present, rabies inactivated vaccine is mainly for dogs and cats over 3 months of age. The immunization period is generally 12 months, and the initial immunization is generally 30-60 days, followed by one strengthening immunization after 28 days. Inactivated vaccine free of adjuvants is for intramuscular injection; however, vaccines containing adjuvants should be injected subcutaneously. The injection dose shall be 
subject to the manufacturers' instructions for the product.

\section{CURRENT SITUATION OF THE USE OF VETERINARY RABIES VACCINE IN CHINA}

At present, the animal rabies vaccine is an inactivated vaccine, which is mainly used for pet dogs and cats in urban and rural domestic situations. However, there is no commercial vaccine for cattle, sheep, foxes, and raccoon dogs that are wild animals. The oral vaccine is expected to be used for wildlife and stray animals in the future, which has been developed by many institutes.

In the past three years, China issued about twentyfive million doses of domestic inactivated rabies vaccine and twelve million doses of imported inactivated rabies vaccine each year, with a total of about thirty-seven million doses. According to preliminary estimates, there are about 110 million dogs and cats in China that means the annual coverage rate of rabies vaccine for dogs and cats in China is only about one-third. In order to effectively control the occurrence of human rabies in China, the animal rabies (especially pet dogs and cats) immunization rate should reach more than $70 \%$; therefore, China needs a large quantity of animal rabies vaccines. At the same time, we should strengthen the development of vaccines suitable for urban and rural stray dogs and cats, as well as foxes, wolves, raccoon dogs and other wild animals, including oral live vaccines and recombinant live vector vaccines and formulate corresponding scientific immunization procedures and application areas. It is suggested that the health, urban management, agriculture, forestry and other related departments in our country cooperate to actively enhance public awareness of rabies and improve the rabies vaccine immunization rate of animals.

doi: $10.46234 / \mathrm{ccdcw} 2021.204$

\# Corresponding author: Chunsheng Yin, zjs62158844@sina.com.

${ }^{1}$ China Institute of Veterinary Drug Control, Beijing, China.

Submitted: August 04, 2021; Accepted: September 22, 2021

\section{REFERENCES}

1. McColl KA, Tordo N, Setién AAA. Bat lyssavirus infections. Rev Sci Tech 2000;19(1):177-96. https://pubmed.ncbi.nlm.nih.gov/1118
$9715 /$.

2. Johnson N, Vos A, Freuling C, Tordo N, Fooks AR, Müller T. Human rabies due to lyssavirus infection of bat origin. Vet Microbiol 2010;142(3-4):151-9. https://pubmed.ncbi.nlm.nih.gov/20188498/.

3. Rupprecht CE, Barrett J, Briggs D, Cliquet F, Fooks AR, Lumlertdacha B, et al. Can rabies be eradicated? Dev Biol 2008;131:95-121. https://pubmed.ncbi.nlm.nih.gov/18634470/.

4. Tordo N, Bahloul C, Jacob Y, Jallet C, Perrin P, Badrane H. Rabies: epidemiological tendencies and control tools. Dev Biol 2006;125:3-13. https://pubmed.ncbi.nlm.nih.gov/16878455/.

5. Bourhy H, Kissi B, Audry L, Smreczak M, Sadkowska-Todys M, Kulonen K, et al. Ecology and evolution of rabies virus in Europe. J Gen Virol 1999;80(Pt10):2545 - 57. http://dx.doi.org/10.1099/00221317-80-10-2545.

6. Liu JJ, Duo L, Tao XY, Zhu WY. Epidemiological characteristics of human rabies in China, 2016-2018. Chin J Epidemiol 2021;42(1): 131 - 6. http://dx.doi.org/10.3760/cma.j.cn112338-20200116-00037. (In Chinese).

7. Feng Y, Wang YY, Xu WD, Tu ZZ, Liu TF, Huo MH, et al. Animal rabies surveillance, China, 2004-2018. Emerg Infect Dis 2020;26 (12):2825-34. https://pubmed.ncbi.nlm.nih.gov/33219645/.

8. Tu C, Feng Y, Wang Y. Animal rabies in the People's Republic of China. Rev Sci Tech 2018;37(2):519-28. https://pubmed.ncbi. nlm.nih.gov/30747129/.

9. Yin CP, Zhou H, Wu H, Shen XX, Wang LH, Yin WW, et al Epidemiological analysis of rabies in 2010, China. Chin J Exp Clin Virol 2011;25(6):434 - 6. http://dx.doi.org/10.3760/cma.j.issn.10039279.2011.06.010. (In Chinese).

10. Zhou GL, Zhao DM, Zhu JJ, Zhao JY, Lin DG, Shi ZS, et al. Evaluation of the effect of rabies vaccines for animals. Chin J Vet Med 2007;43(4):50 - 3. http://dx.doi.org/10.3969/j.issn.0529-6005.2007. 04.026. (In Chinese).

11. Shi HH, Song WK, Huang YL, Liu XY, Dai YY, Fu QL, et al. Efficacy of protection effect induced by inactivated rabies vaccine (Hep-FlurydG). Chin J Zoon 2012;28(7):664 - 8. http://dx.doi.org/10.3969/cjz. j.issn.1002-2694.2012.07.004. (In Chinese).

12. Lin F, Zhu JH, Chen NM, Hou YD, Esposito J. Construction and characteristic of vaccinia virus recombinants expressing rabiesvirus glycoprotein. Chin J Virol 1992;8(3):210-7. https://www.docin.com/p$881933015 . \mathrm{html} \& \mathrm{dpage}=1$ \&key $=\% \mathrm{E} 7 \% 8 \mathrm{~B} \% 82 \% \mathrm{E} 7 \% 8 \mathrm{~A} \% \mathrm{AC} \% \mathrm{E} 7 \%$ $97 \% 85 \% \mathrm{E} 6 \% 80 \% 8 \mathrm{E} \% \mathrm{E} 4 \% \mathrm{~B} 9 \% 88 \% \mathrm{E} 6 \% \mathrm{~B} 2 \% \mathrm{BB} \&$ is Pay $=-$ $1 \&$ toflash=0\&toImg=0. (In Chinese).

13. Zhao WG, Ying BL, Wang JL, Cai B, Zhu YT, Hu QL, et al. Rabies virus $\mathrm{N}$ protein expressed by recombinent vaccinia virus and the observation of its immunoeffect. Chin J Exp Clin Virol 1993;7(1):60 4. http://dx.doi.org/10.3760/cma.j.issn.1003-9279.1993.01.121. (In Chinese).

14. Li WH, Zhang Y, Wang SH, Liu L. Immune response of mice to replication-defective recombinant adenovirus containing glycoprotein gene of rabies virus 3aG strain. Chin J Exp Clin Virol 2001;15(1):61 5. http://dx.doi.org/10.3760/cma.j.issn.1003-9279.2001.01.015. (In Chinese).

15. Zhang SF, Hu RL, Xiao YQ, Tian XY, Xia XZ. Construction of recombinant canine adenovirus type-2 expressing rabies virus glycoprotein. Virol Sin 2005;20(2):155-8. https://d.wanfangdata.com. cn/periodical/ChlQZXJpb2RpY2FsQ0hJTmV3UzIwMjEwOTA5Eg56 Z2JkeDIwMDUwMjAxMxoIYzlnNWUxYTY\%3D. (In Chinese).

16. Wang Y, Zhang SF, Liu Y, Zhang F, Zhang JX, Hu RL. Generation and preliminary immunological efficacy of a recombinant human adenovirus-rabies virus glycoprotein. Chin J Virol 2011;27(5):442 - 6 . http://dx.doi.org/10.13242/j.cnki.bingduxuebao.002203. (In Chinese).

17. Xiang ZQ, Ertl HCJ. A simple method to test the ability of individual viral proteins to induce immune responses. J Virol Methods 1994;47 (1 - 2):103 - 16. http://dx.doi.org/10.1016/0166-0934(94)90070-1.

18. Wu QF, Yu FL, Xu JF, Li Y, Chen HC, Xiao SB, et al. Rabies-virusglycoprotein-pseudotyped recombinant baculovirus vaccine confers complete protection against lethal rabies virus challenge in a mouse model. Vet Microbiol 2014;171(1 - 2):93 - 101. http://dx.doi.org/ 10.1016/j.vetmic.2014.03.037. 